Document downloaded from:

http://hdl.handle.net/10251/63114

This paper must be cited as:

Cervelló Royo, RE.; Guijarro Martínez, F.; Michniuk, K. (2015). Stock market trading rule based on pattern recognition and technical analysis: Forecasting the DJIA index with intraday data. Expert Systems with Applications. 42(14):5963-5975. doi:10.1016/j.eswa.2015.03.017.

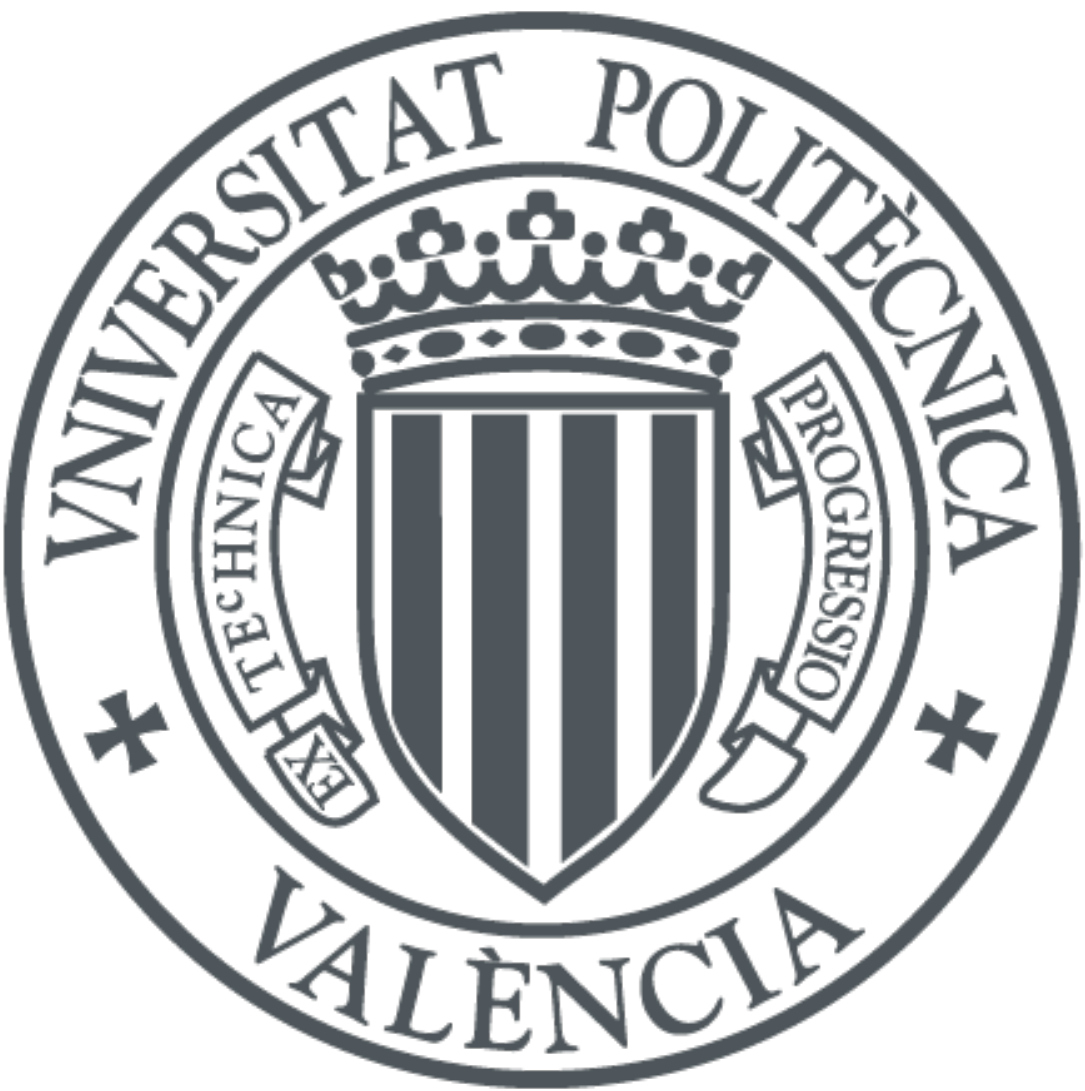

The final publication is available at

http://dx.doi.org/10.1016/j.eswa.2015.03.017

Copyright Elsevier

Additional Information 


\title{
Stock market trading rule based on pattern recognition and technical analysis: forecasting the DJIA index with intraday data
}

\author{
Authors \\ Roberto Cervelló-Royo \\ Faculty of Business Administration and Management, Universidad Politécnica de Valencia, \\ Camino de Vera s/n, 46022, Valencia, tel.963877007 ext.74710; rocerro@esp.upv.es \\ Francisco Guijarro* \\ Faculty of Business Administration and Management, Universidad Politécnica de Valencia, \\ Camino de Vera s/n, 46022, Valencia, tel.963877007 ext.88204; fraguima@esp.upv.es \\ Karolina Michniuk \\ Faculty of Business Administration and Management, Universidad Politécnica de Valencia, \\ Camino de Vera s/n, 46022, Valencia and \\ Faculty of Business \& Social Sciences, Hamburg University of Applied Sciences, Berliner Tor 5, \\ 20099 Hamburg; Karolina.Michniuk@ haw-hamburg.de
}

*Corresponding author 


\title{
Stock market trading rule based on pattern recognition and technical analysis: forecasting the DJIA index with intraday data
}

\author{
Authors \\ Roberto Cervelló-Royo \\ Francisco Guijarro \\ Karolina Michniuk
}

\begin{abstract}
This study introduces a new approximation of the flag price pattern recognition. We develop a trading rule which provides positive risk-adjusted returns for intraday data of the Dow Jones Industrial Average Index. In order to mitigate the data snooping problem we use a data set of more than 90,000 observations, results are reported over 96 different configurations of the trading rule parameters. Furthermore, results are examined over 3 non-overlapping sub-periods. The trading rule provides positive results for all the configurations.
\end{abstract}

Keywords:

Technical Analysis

Pattern recognition

Stock Market Trading Rule

Forecasting

Financial Expert Systems

Intraday Data

\author{
Abbreviations: \\ DJIA (Dow Jones Industrial index) \\ SL (Stop Loss) \\ TP (Take Profit) \\ Thr (Threshold fitting value) \\ \#Ops (Number of operations) \\ \#PosOps (Number of positive operations) \\ \#NegOps (Number of negative operations) \\ $\mathrm{HR}$ (Hit ratio $=$ \#PosOps / \#Ops) \\ TR (Total Return) \\ AR (Average of the returns) \\ MD (Maximum Drawdown) \\ $\mathrm{TR}>\mathrm{MD}$ (is TR greater than MD?) \\ VAR (value at risk) \\ SPA (Superior Predictive Ability)
}




\section{Introduction}

Technical analysis has attracted attention from both professionals and academics in the latter decades. The first conceived works validated the efficient nature of the market and therefore that, from the historic price series, it was not possible to infer any profitable strategy more efficient than the market (Alexander, 1961; Jensen and Bennington, 1970).

Recently, some authors have found positive empirical evidence over the return of simple trading rules based on moving averages, support and resistance, channels, or visual chart patterns. For further details see Park and Irwin (2007).

Neftci (1991) concludes that the rules of technical analysis might capture some information ignored by Wiener-Kolmogorov prediction theory. His results over the Dow Jones Industrial index (DJIA) for 1911-1976 suggested that this is the case for the moving average trading rule. In line with this, Brock et al. (1992) confirm that returns obtained from technical trading rules are not consistent with four common models in finance theory: the random walk, the AR(1), the GARCH-M, and the Exponential GARCH. Ratner and Leal (1999) state that 82 out of the 100 country-trading rule combinations tested in ten emerging markets, disregarding their statistical significance, correctly predict the direction of changes in the return series.

White (2000) alerts about some problems which had arisen from the choice of trading rules whose performance had been positive in the past. Any positive results obtained may be due to chance rather than to any merit inherent in the method. The choice of the same data both for the election of the model and for its later validation is called data snooping, a problem already remarked by Brock et al. (1992). White (2000) proposes the use of the reality check in order to mitigate the problems of data snooping; however, Hansen (2005) shows that those results might be sensitive to the inclusion of irrelevant alternatives.

It is our belief that some of the contradictory results regarding the performance of technical analysis are due to the use of extremely simple trading rules. Some of these works conclude that technical analysis does not provide an added value to the stock market decisions. The authors state that those simple trading rules are not significant after considering the effect of data snooping. However, it is difficult to consider that investors will make their decisions by using only a simple moving average, or a combination of supports and resistances. Furthermore, the validity of the technical analysis can not be completely rejected because some trading rules have not provided positive results, with independence of several of the configurations of the trading rules that have been tested. It seems reasonable to think that decision making in trading is a more complex process, in which a major number of factors take part, where different technical indicators and trading rules can be combined and more than one timeframe can be considered. This more complex and dynamic vision is defined as romantic by Leigh, Purvis et al. (2002), who considered that the romantic style is data-driven, rather than theory-driven.

In this vein, there is abundant literature related to the chart pattern recognition. For example, Lo et al. (2000) propose a systematic and automatic approach using nonparametric kernel regression. They apply this method to a large number of U.S. stocks and find that over the 31year sample period, several technical indicators provide incremental information.

One of the chart patterns which academics have paid more attention to is the flag pattern. Leigh, Paz et al. (2002), Leigh, Purvis et al. (2002), Leigh et al. (2004) and Wang and Chan $(2007,2009)$ have reported positive performance of trading rules based on the flag pattern by employing different stock market indexes and for a relatively wide period of time. The profitability of this trading rule has been greater than the index selected as a benchmark, even after including the transaction costs.

In order to test the statistical significance of these results, and to mitigate the effects of data snooping, attention should be paid to what Brock et al. (1992) remark: (1) to report results from all trading rules, (2) to use very long data series, and (3) to emphasize the robustness of results across various non-overlapping sub-periods.

The aim of this work is to follow the line of research started with the graphical patterns, by proposing a new version of the flag pattern which includes parallelisms with the rule IF-THEN. The validation of the trading rule also presents important novelties with respect to previous works: (1) two new parameters are included, the stop loss and the take profit, which allows 
modeling of the closing of operations in a dynamic way; (2) intraday data are employed, which allows considerable width in the number of observations in the sample; (3) not only closing prices are considered, but also opening prices (Japanese candlesticks body); thus, the information considered when deciding whether or not to start an operation is widened. Besides evaluating the performance of the trading rules through its profitability, we also contemplate the risk by considering the maximum drawdown of the return curves, since the non normality of the returns prevents us from using the t-student.

The results confirm the positive performance of the flag pattern over the intraday data of the DJIA for a time horizon of more than 13 years, opposite to Marshall et al. (2008) who did not find any positive empirical evidence for the US equity market during a period of only 2 years.

The rest of the work is structured as it follows: in the next section the weight matrix that identifies the flag pattern, linking the rule IF-THEN, is presented; in the third section the trading rule is develop, and the stop loss and take profit values are defined according to the price range; in the fourth section we present the results obtained when applying the trading rule over the intraday data of the Dow Jones Industrial Average (DJIA); the fifth section presents the results overt the 3 non-overlapped sub-periods; finally, the last section summarizes the findings and offers conclusions.

\section{Flag pattern recognition}

Charting analysis is based on the recognition of certain graphical patterns in the price and, eventually, in the volume of operations. This work focuses on the graphical analysis of prices, identifying one of the more recognized and analyzed patterns in the academic literature: the flag. A definition of this pattern can be found in Downes and Goodman (1998): "Technical chart pattern resembling a flag shaped like a parallelogram with masts on either side, showing a consolidation within a trend. It results from price fluctuations within a narrow range, both preceded and followed by sharp raises or declines".

To the best of our knowledge, the works by Leigh, Modani et al. (2002), Leigh, Paz et al. (2002) and Leigh, Purvis et al. (2002) are the first ones to deal with the graphical recognition of this kind of pattern and to introduce a trading rule linked to it. In order to identify the pattern, we use a 10x10 grid of weights, like the one that figure 1 shows, which allows to recognize the bull flag pattern (Leigh, Purvis et al., 2002).

\section{[Here Figure 1]}

The first 7 columns of the weights matrix represent the Downes and Goodman consolidation process, whereas the last 3 columns represent the breakout (sharp increase of the price). The bear flag variation would be obtained as a mirror reflection over the horizontal axis.

In order to check if the price series show a flag or not, we fit the template over the price window we want to check; thus, the highest price in the window is made to correspond with the top of the grid, and the lowest price in the window is made to correspond with the bottom of the grid. The difference between the maximum and the minimum constitutes the price range $(R)$ or height of the window. The goal of this process is to obtain the fit value that will inform the level of matching between the matrix and the price window: when the price in a determined instant $t$ falls in a cell labeled 1, the fit value will increase in a unit; if the price falls in a cell labeled 0.5 , the fit value will increase in 0.5 units; this is done for each one of the different values that appear in the template of figure 1 and each one of the instants $t, t=1 . .10$.

Since each element of the price window can only coincide with one cell of the grid (in cited literature, authors work with closing prices), a window with 10 prices will restrict to 10 the maximum value of the fitting function and this total fitting value will provide the maximum fitting of the price window to the flag pattern. In the most common case, in which prices will not only fall in cells labeled 1, the total fitting value will be below 10, the greater difference between the price window and the bull flag template (figure 1) the lower the total fitting value. The fitting value threshold (minimum total fitting value) should be established by the researcher 
or the trader a priori. A very demanding threshold will reduce the number of identified flags, restricting with this the statistical significance of the results; while a more permissive one might take as flag pattern some window prices, which hardly should be considered in other case.

The determination of the weights can result in a black box for the users (Wang y Chan, 2007). Like Zapranis and Tsinaslanidis (2012) state, "in template matching techniques the user has to set specific weights in the template's grid before the identification of a pattern. These weights in some cases are set somehow arbitrarily and the process embeds a level of subjectivity".

The choice of the threshold value is closely related to the grid of weights, and the choice of these weights is critical when identifying the pattern. Figure 2 presents two window prices which, considering the grid of weights of figure 1, provide the same total fitting value of 6.5. While in the first case (figure 2.a) it can be assumed that the price window corresponds to a bull flag pattern, in the second case (figure 2.b) the price goes down without confirming a following breakout and the shape deviates far from a bull flag pattern. One solution might be to increase the threshold of the total fitting value, but in this case we would discard price windows that will fit the pattern (figure 2.a).

\section{[Here Figure 2]}

This work tries to mitigate this inconvenient by making an alternative choice of weights. Figure 3 contains the proposed grid of weights that will allow to identify a bull flag pattern.

\section{[Here Figure 3]}

The first difference with respect to the matrix of figure 1 lies in the shape of the bull flag pattern. While Leigh, Modani et al. (2002), Leigh, Paz et al. (2002), Leigh, Purvis et al. (2002) y Wang and Chan $(2007,2009)$ use the consolidation and breakout version, we explore the breakout and consolidation approach. The second difference lies in the range of weights considered and in the distribution of the weights around the matrix: the weights configuration is a key point in our proposal.

The matrix of figure 3 contains an only cell with positive value, which points the area from which the price should start: left bottom corner. It should be noted that in order to get a strictly positive value for the fitting function, the price must pass through this cell. The cells with negative values indicate areas in which the price should not fall, in case we want the price window to be considered a bull flag pattern. Whereas the cells with value 0 , mark the areas where the price could move without affecting the total value of the fit function.

This configuration of weights identifies the pattern more definitively in comparison to other kinds of grid, since other grids might consider that considerable different price windows adopt the shape of a flag (figure 2). Our proposal is more related to an IF-THEN rule. For example, if we decide that those price windows whose fitting value is greater or equal to 4 are the only ones to be considered flags, then the following conditions must take place:

Price should fall in the cell labeled 5.

Price should visit one cell with negative weight at the most.

These two conditions strictly limit the cells in which price can fall, thus at least 8 of the 10 price columns must fall in the cells labeled 0 . If both conditions are fulfilled (IF) then the price window is considered a bull flag pattern (THEN).

Another important difference with respect to other works is the matching between the historical prices and the grid of weights; we do not take the closing prices, but the body of the candlesticks. This implies that a candlestick can fall in more than one cell per column. Therefore, the fitting function will not be found by only adding the value of the 10 cells, since it could include a greater number.

In order to include more information about prices evolution, we have adopted the candlesticks body variation instead of the more simple closing price. The use of the Japanese candlesticks in charting analysis has become widespread; in fact, currently most of the trading platforms work with this kind of representation by defect. We have not used the total range of 
the candlestick, high and low difference, since these extremes represent price levels which have already been reached after the opening and rejected before the closing.

\section{Trading rule specification}

In the previous section, we have defined the way in which the flag patterns are identified. The following step will consist in defining the trading system. According to Park and Irwin (2007) a technical trading system consists of a set of trading rules that generate trading signals according to various parameter values. In order to implement a trading system it is necessary to determine the starting moment of a buying (selling) operation of an asset, and the conditions required to close the operation.

Once the proper matching is obtained from the comparison between the price window and the grid of weights, the buying or selling operation is started depending on the bull or bear flag recognition. Therefore, if the matching takes place for the price window between $t$ and $t+9$, the operation will start with the opening price in $t+10$.

In order to evaluate the return of the operation, the exit point should be defined. Most of the literature considers a holding period of $d$ candlesticks. The value $d$ will change according to different authors: $d=6$ in Lee and Jo (1999), $d=20$ in Leigh, Purvis et al. (2002) and $d=100$ in Leigh, Modani et al. (2002). In order to increase the solidity of the results and mitigate the data snooping effect some authors propose to consider a group of values for $d$ instead of an only one: $d \in\{10,20,40,80$,$\} in Leigh, Paz et al. (2002),$ $d \in\{20,40,60,80,100\}$ in Leigh et al. (2004) and in Wang and Chan (2007), and $d \in$ $\{20,40,60,80,100,120,160,200,240\}$ in Wang and Chan (2009).

An interesting alternative won't be statically fixing a determined value or group of values, but instead adopting a dynamic process in which operations will close depending on the evolution of the price, and not on the time. Teixeira and Oliveira (2010) propose the use of a variation often used by traders: to put a stop loss and a take profit in each operation, which will limit both the loss and the profit of each one of the operations. Once a position is initiated, the stop loss will mark the price level at which the maximum supported loss will be reached, thus if prices reach the stop loss then the operation will close assuming that loss. In the same way, the take profit will mark the price level at which the expected profit or target of the operation should be taken, thus when the price reaches this level the operation will close and provide the corresponding profit.

As a general rule, the gain at the take profit level usually is greater than the loss at the stop loss level. This makes the resulting average profit by operation greater than the experimented average loss, thus the total profit will depend on the success ratio of the operations.

In our case, we have defined the stop loss and the take profit in a relative way with respect to the price range $R$ of the pattern. That is to say, if the flag has been developed over a narrow range of prices, then the stop loss and the take profit will also be small. In the case the flag had been developed over a wide range of prices, the stop loss and the take profit will be relatively big.

Figure 4 contains an illustrative example for 15 minutes candlesticks over the DJIA futures, from 5:00 p.m. to 10:00 p.m. on June $16^{\text {th }}, 2003$. Image shows a) a bull flag identification and b) the stop loss and the take profit depending on the price range $R$. In this case, the matching among the 10 first candlesticks and the grid of weights provides a fitting value of 5 , the maximum we can get. We have set a stop loss of 0.5 and a take profit of 1 over the price range $R=48$. Since the opening price of the 11 th candlestick is 9249 , the stop loss will be at the level $9249-0.5 * 48=9225$ and the take profit will be at the level $9249+1 * 48=9297$. The 9:45 p.m. candlestick reaches the take profit, thus the full operation will provide a positive result of 48 points. 


\section{Results}

According to the trading rule introduced in the previous section we have chosen the future of one of the most internationally well known indexes: the DJIA. In order to get a historical series wide enough to allow to infer significant results and to mitigate the data snooping effect, we select a 15 minutes timeframe during the period which lasts from May $22^{\text {nd }} 2000$ to November $29^{\text {th }}$ 2013. During this period, there have been bullish, bearish and lateral market stages. The total number of candlesticks amounts to 91,307.

Most authors use daily data, which 1) limits the number of observations and 2) demands taking a very delayed historical price series in order to reach a representative number of observations. The use of previous historical data with respect to the current moment can presume a problem when trying to validate a trading rule: "Suppose that some technical trading rules can be found that unambiguously outperform the benchmark over the sample period, but that these are based on technology (e.g., neural networks) that only became available after the end of the sample. Since the technique used was not available to investors during the sample period, we do not believe that such evidence would contradict weak-form market efficiency" (Sullivan et al., 1999). In this line of argument Timmermann and Granger (2004) question the use of strategies designed over the historical data in the short term, since once the more recent information is included, the price strategy stops being successful.

The use of intraday data does not affect the price pattern identification, due to the fractality which underlies the stock market data. Elder (2002), one of the most reputed traders, states "if you remove price and time markings from a chart, you won't be able to tell whether it is weekly, daily, or intraday. Markets are fractal". Also Bollinger (2002), who introduced the Bollinger bands, defends the existence of fractality at financial markets: "It turns out that fractal patterns are very common. For example, take a long-term W bottom. When examined closely, the W may turn out to have intermediate-term W bottoms embedded in its footings"

Given a price range $R$, the multiples considered in order to fix the stop loss $(S L)$ and the take profit $(T P)$ have been:

$S L \in\{0.2,0.4,0.6,0.8\}$

$T P \in\{1.0,1.2,1.4,1.6,1.8,2.0\}$

The combination of these values will allow to work with 24 different stop loss and take profit configurations.

The threshold fitting value is also a configurable parameter:

Threshold fitting value $\in\{2,3,4,5\}$

Therefore, the total number of configurations amounts to 96. For each one of them we have calculated the total number of operations, the number of successful operations, the number of failed operations, the hit ratio, the total return, the average of the returns and the maximum drawdown. Table 1 summarizes the results ${ }^{1}$.

\section{[Here Table 1]}

As expected, the number of operations is inversely proportional to the threshold fitting value $(T h r)$. In the most selective case $T h r=5$ only 483 flag patterns were identified, which a $0.53 \%$ of the total sample. In the case $T h r=2$ the number of identified patterns is 1,402 ; that is to say, $1.54 \%$ of the sample. Although they can seem low significant percentages, we should highlight that the flag is one pattern among a multitude of technical figures that can appear on the charts. 
If we considered the whole universe of technical figures, the percentage would increase reasonably. However, it is reasonable to think that the market does not offer investment opportunities in a permanent way, but these will present from time to time and they will usually be linked to relevant news or information.

By analyzing the trading rule performance we can check how the total return $T R$, total sum of the operation returns, is positive in the 96 cases; the same thing happens with the average of returns $A R$. The maximum return $180.2 \%$ is obtained by the configuration $T h r=2, S L=0.2$ and $T P=2$. In the worst case scenario, a total return of $28.8 \%$ is obtained, by combining $T h r=3, S L=0.8$ and $T P=1$. For this configuration, we also obtained the worst average of returns $(0.18 \%)$ by the combination $T h r=5, S L=0.6$ and $T P=1.8$.

When comparing to the benchmark, the DJIA, and for the considered period, we obtained a average of returns of 0.0000042 . Therefore, all the configurations have provided a higher average of returns than the DJIA index.

Viewing the results, we can conclude that the trading rule gets more positive and higher results than the benchmark. The only requirement that an investor should consider would be that the transaction costs should not exceed the obtained average of returns for the different configurations of Table 1 .

The percentage of successful operations is always lower than the failed operations $(H R<50 \%)$, this is in accordance with the imposed restriction that take profit should be greater than the stop loss. However, as it has been already commented, the total return and the average of returns are positive in all cases.

Figure 5 shows the total return curves for different configurations of the trading rule; moreover, all the considered threshold levels have been included.

\section{[Here Figure 5]}

Besides the return, we have also estimated the risk for each one of the configurations. The non normality of the trading rule returns impedes the application of the statistical $t$ (Leigh et al., 2004) and the estimation of the mean return intervals. In the present study, the non normality of the returns is even higher than in other works, due to the levels of stop loss and take profit we have chosen. The restriction that the first one must be lower than the second one signifies that the returns follow a clearly asymmetric distribution, as we can infer from the hit ratio in Table 1 .

The non-normality of returns made us look for an alternative which could help measure the risk of the strategy: the maximum drawdown $(M D)$.

The drawdown at moment $t, D_{t}$, is defined as the drop of the return curve from the previous maximum at $s, s<t$. For instance, if at the moment $s$ the total return $(T R)$ curve reaches a new maximum of $60 \%$, and after several operations the total return at $t$ drops to $45 \%$, then the drawdown at moment $t$ would be $15 \%$.

$$
\begin{gathered}
D_{t}=\max _{s<t} T R_{s}-T R_{t} \\
M D=\max _{t} D_{t}
\end{gathered}
$$

The maximum drawdown corresponds with the maximum loss experienced during all the period, keeping certain similarity with the value at risk (VAR). For instance, considering the configuration with the greatest total return $180.2 \%$ we have estimated a maximum drawdown of $11.5 \%$. This value indicates that if we had started the trading rule at the worst moment, the maximum loss we could support would be $11.5 \%$.

In the last column of table 1 we can check that the total return is greater than the maximum drawdown in 95 of the total 96 configurations. Therefore, the use of this trading rule would have guaranteed a greater profit than the risk supported in a $98.96 \%$ of total configurations. 
In order to compare with the DJIA, its drawdown was $78.42 \%$ and the total return was $37.53 \%$ during the whole period. Therefore, the risk-adjusted return of the trading rule is better than the risk-adjusted return of the DJIA.

The relation between the trading rule performance and the parameters of its configuration has been also studied (table 2).

It is worth noting the correlation between the threshold and both the variables total return and the average of returns. The total return is negatively related to the threshold level $(-0.70)$, while the average of returns per operation is positively related (0.50). It means there is a positive relation between the return of the operations and the similarity which exists between the price window and the flag pattern. Therefore, the more recognizable and clearer the pattern of a flag is, the greater the return of the trading rule. The negative relation between the threshold and the total return is explained by the greater number of operations for low threshold values. In fact, the correlation between the threshold and the total return after removing the effect of the number of operations is not statistically significant, with a coefficient value of -0.08 and a $p$ value of 0.42 .

It is also interesting to check how both the total return and the average of returns are negatively related to the stop loss and positively related to the take profit. This confirms the levels choice of take profit, which are greater than the stop loss when defining an investment strategy.

With respect to the risk, the maximum drawdown is negatively related to the average of returns $(-0.52)$. This would contradict the fundamentals of financial markets: return and risk are positively related. In order to contrast this hypothesis and once we have removed the effect of the number of operations, the correlation coefficient and the p-value we found were 0.01 and 0.90 respectively. Therefore, the number of operations will explain the relation between the average of returns and the maximum drawdown.

To summarize, a greater level of threshold will involve a greater average of returns, without assuming a greater risk. That is to say, the flag pattern provides a positive and significant riskadjusted return.

[Here Table 2]

\section{Analysis of non-overlapped periods}

Brock et al. (1992) state that the data snooping problem can be mitigated by using (1) a very long data series, (2) reporting results from all trading rules, and (3) providing results across various non-overlapping sub-periods. In the present work and thanks to intraday data we have used a very long historical data series. We have also configured the trading rule in order to analyze its behavior over different and plausible configurations. Therefore, it only remains the use of several non-overlapping sub-periods.

This section presents the results of applying the trading rule over 3 non-overlapping subperiods; all of them are of the same size with nearly 31,500 15 minutes candlesticks. The first sub-period lasts from May $22^{\text {nd }} 2000$ to November $26^{\text {th }} 2004$; the second sub-period lasts from November $26^{\text {th }} 2004$ to February $27^{\text {th }} 2007$; and the third sub-period lasts from February $27^{\text {th }}$ 2007 to November $29^{\text {th }} 2013$. The aim is to check if the behavior of the trading rule for each one of the sub-periods is similar to the one observed for the whole sample.

For this purpose we have again applied the 96 configurations of the trading rule over the 3 sub-periods (results are presented in table 3). With the aim of reducing the length of the study, the information about the total return, the average of returns and the maximum drawdown are the only ones presented.

It can be noticed that the total return results positive in all cases for sub-period 1. Moreover, in most of the configurations the maximum drawdown is lower than the total return for the whole sub-period. 
Sub-period 2 has a very similar behavior to sub-period 1 . Total return results positive in all instances, and only in a few cases did the maximum drawdown exceed the total return.

Sub-period 3 results are more complex. Total return is negative for 12 configurations, and 34 configurations provide a maximum drawdown which is greater than the total return.

Therefore, it can be concluded that 2 of 3 sub-periods perform in a similar way to the entire sample, whereas the third sub-period presents some cases of negative returns and risks greater than the return.

Furthermore, the lower the values of the stop loss the better the performance of the trading rule. When this parameter takes a value of 0.2 , we can check how the total return is positive for all sub-periods and the rest of parameter values; and also how total return is greater than the maximum drawdown. A similar conclusion is reached when taking a value of 0.4 , all the total returns are positive and only in some cases the risk is greater than the return. These results are consistent with the correlations showed in table 2, where we can confirm that low levels of stop loss favored a greater return and a lower level of drawdown. In fact, in table 2 it can be checked that the greater correlation coefficient in absolute value relates the stop loss and the maximum drawdown.

\section{[Here Table 3]}

\section{Conclusions}

This study confirms the positive results obtained by other researchers about the trading rule based on flag pattern recognition, by adding some relevant changes. Firstly, we have used the breakout and consolidation flag pattern version, rather than the consolidation and breakout version which has already been examined in the literature. A new definition of the grid of weights allows us to relate this kind of patterns to the IF-THEN rule, which we understand to be closer to the decision making of the stock market investors. Furthermore, this new approach reinforces the positive results reported on the flag pattern in previous studies. Secondly, in order to address the data snooping problem, different topics have been considered: the first one was linked to the use of intraday data, which allowed us to use historical data series of more than 90,000 observations. The second one introduces a dynamical approach when specifying the trading strategy, by closing the operations when the price level reached the stop loss or take profit. The definition of these two parameters together with the threshold, which limits the fitting of the price series to the flag pattern, allowed us to consider 96 different configurations. The third topic was to validate the trading rule over 3 non-overlapping sub-periods.

We have confirmed how the trading rule provided positive results for all the configurations, with a generally positive performance for the three sub-periods. Results gathered from the whole sample confirm that the trading rule provides a positive return, even after considering the risk. Moreover, it beats the benchmark in the mean variance sense. The only negative consideration that an investor should contemplate would be to avoid transaction costs exceeding the average of returns obtained with the strategy.

The best results have been obtained when considering adjusted levels of stop loss, between 0.2 and 0.4 times the price rank; and also for the high values of take profit with respect to the given stop loss. The explanation of those stop loss levels can be found when defining the grid of weights which identifies the flag pattern. The proposed weights clearly limit one consolidation area which the price should not pass through, this area is located over the $30 \%$ limit (three first rows of the figure). In fact, this level corresponds to a stop loss of $0.2 / 0.4$, where the strategy gets its best results. This result indicates that this level is acting as "support" of those operations, thus when the price passes through this level, it will be quite likely to finish the operation with losses, whereas when the price stays out of this level, it will be very likely to succeed in the operation.

Future lines of research might deal with the application of this methodology over the intraday data of other indexes or financial assets, the definition of the grid of weights whose 
numbers of columns are different to 10 or the application of White's reality check or the Hansen SPA in order to even more limit the data snooping effect.

\section{References}

Alexander, S. (1961). Price movement in speculative markets: trends or random walks. In P. Cootner (Eds.), The random character of stock market prices (pp. 199-218). Cambridge, Mass: MIT Press.

Bollinger, J. (2002). Bollinger on Bollinger Bands. New York: McGraw Hill.

Brock, W., Lakonishok, J., LeBaron, B. (1992). Simple technical trading rules and the stochastic properties of stock returns. Journal of Finance, 47(5), 1731-1764.

Downes, J., Goodman, J. (1998). Dictionary of finance and investment terms, (5th ed.). New York: Barron's Educational Series, Inc.

Elder, A. (2002). Come Into My Trading Room: A Complete Guide to Trading. New York: John Wiley \& Sons Inc.

Hansen, P. (2005). A test for superior predictive ability. Journal of Business and Economic Statistics, 23(4), 365-380.

Jensen, M., Bennington, G. (1970). Random walks and technical theories: some additional evidences. Journal of Finance, 25(2), 469-482.

Lee, K., Jo, G. (1999). Expert system for predicting stock market timing using a candlestick chart. Expert Systems with Applications, 16(4), 357-364.

Leigh, W., Modani, N., Hightower, R. (2004). A computational implementation of stock charting: abrupt volume increase as signal for movement in New York Stock Exchange Composite Index. Decision Support Systems, 37(4), 515-530.

Leigh, W., Modani, N., Purvis, R., Roberts, T. (2002). Stock market trading rule discovery using technical charting heuristics. Expert Systems with Applications, 23(2), 155-159.

Leigh, W., Paz, N., Purvis, R. (2002). Market timing: a test of a charting heuristic. Economics Letters 77(1), 55-63.

Leigh, W., Purvis, R., Ragusa, J. (2002). Forecasting the NYSE composite index with technical analysis, pattern recognizer, neural network, and genetic algorithm: a case study in romantic decision support. Decision Support Systems, 32(4), 361-377.

Lo, A., Mamaysky, H., Wang, J. (2000). Foundations of technical analysis: computational algorithms, statistical inference, and empirical implementation. Journal of Finance, 55(4), $1705-1770$.

Marshall, B., Cahan, R., Cahan, J. (2008) Does intraday technical analysis in the US equity market have value? Journal of Empirical Finance, 15, 199-210.

Neftci, S. (1991). Naive trading rules in financial markets and Weiner-Kolmogorov prediction theory: a study of technical analysis. Journal of Business, 64(4), 549-571.

Park, C., Irwin, S. (2007). What do we know about the profitability of technical analysis. Journal of Economic Surveys, 21(4), 786-826.

Ratner, M., Leal, R. (1999). Test of technical trading strategies in the emerging equity markets of Latin America and Asia. Journal of Banking and Finance, 23(12), 1887-1905.

Sullivan, R., Timmermann, A., White, H. (1999). Data snooping, technical trading rule performance, and the bootstrap. Journal of Finance, 54(5), 1647-1692.

Teixeira, L., de Oliveira, A. (2010). A method for automatic stock trading combining technical analysis and nearest neighbor classification. Expert Systems with Applications, 37(10), $6885-6890$.

Timmermann, A., Granger, C. (2004). Efficient market hypothesis and forecasting. International Journal of Forecasting, 20(1), 15-27.

Wang, J., Chan, S. (2007). Stock market trading rule discovery using pattern recognition and technical analysis. Expert Systems with Applications, 33(4), 304-315.

Wang, J., Chan, S. (2009). Trading rule discovery in the US stock market: An empirical study. Expert Systems with Applications, 36(3), 5450-5455.

White, H. (2000). A reality check for data snooping. Econometrica, 68(5), 1097-1126. 
Zapranis, A., Tsinaslanidis, P. (2012). A novel, rule-based technical pattern identification mechanism: Identifying and evaluating saucers and resistant levels in the US stock market. Expert Systems with Applications, 39(7), 6301-6308. 